\title{
A basic mathematical testbed for energy efficiency analyses of DC power distribution systems/microgrids
}

\author{
Faizan Dastgeer ${ }^{1}$, Hafiz Muhammad Anees ${ }^{2}$, Hasan Erteza Gelani ${ }^{3}$, Kashif Amjad ${ }^{4}$, Rameez Arif ${ }^{5}$ \\ ${ }^{1,3,5}$ Department of Electrical Engineering, University of Engineering and Technology Lahore-FSD Campus, Faisalabad, \\ Pakistan \\ ${ }^{2}$ Karachi Institute of Power Engineering (KINPOE), Pakistan Institute of Engineering and Applied Sciences (PIEAS), \\ Islamabad, Pakistan \\ ${ }^{4}$ Department of Electrical Engineering, National University of Computer and Emerging Sciences, Chiniot-FSD Campus, \\ Faisalabad, Pakistan
}

\section{Article Info \\ Article history: \\ Received Jan 2, 2021 \\ Revised Apr 26, 2021 \\ Accepted May 7, 2021

Keywords:
DC distribution networks
DC microgrids
DC vs. AC
Efficiency
Energy savings \\ Keywords: \\ DC distribution networks \\ Efficiency

\begin{abstract}
DC power distribution has become a topic of interest in the recent past, despite being given up a long time. A number of research efforts have been carried out in this field; especially the idea of DC distribution for microgrids has witnessed a significant amount of time and attention. System efficiency is one of the under-research areas of this field that has witnessed a lot of research efforts which were generally simulation based studies. Detailed mathematical efficiency analysis is missing in the present body of knowledge. In this regard, the current research effort aims to present a foundation level mathematical efficiency analysis set-up that may be used as an analytical testbed for different efficiency studies especially those related to the comparative evaluation of DC and AC systems. The current effort highlights the strong dependence of $\mathrm{AC}$ and $\mathrm{DC}$ distribution system efficiency on the factor of time. The efficiency advantage of DC over AC or vice versa within a specified duration depends on the ratio of $\mathrm{DC}$ and $\mathrm{AC}$ grid powers summed over the duration.
\end{abstract}

This is an open access article under the CC BY-SA license.

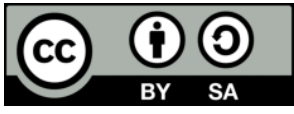

Corresponding Author:

Kashif Amjad

Department of Electrical Engineering

FAST National University of Computer and Emerging Sciences

Chiniot Faisalabad Campus, 35400, Punjab, Pakistan

Email: kashif.amjad@nu.edu.pk

\section{INTRODUCTION}

Direct current (DC) for the transfer of electrical energy-this is how the electric power system started [1], [2]. However, this was given up soon and alternating current (AC) was adopted which remained as a standard practice for more than a century-and enjoys this place even now. Nevertheless, DC has reinstalled itself in the electric power system in the form of high voltage DC (HVDC) transmission lines, modern electronic loads and solar photovoltaic energy generation; while it is in the research phase for being used in residential distribution systems [3]-[5] and microgrids [6]-[9].

Efficiency is a concept having a long and strong relationship in the DC vs. AC battle. Why DC was given up? Because AC systems provided higher power/energy efficiency. And why DC was re-introduced? Apparently, this was again due to the higher energy efficiency of DC power transmission as compared to AC for under-sea cables for the Gotland project in Sweden in 1954 when the first commercial HVDC line was installed. Afterwards, the HVDC technology witnessed further development and it was proven to deliver higher efficiency as compared to AC, provided certain condition(s). Consequently, these lines have been 
installed in various places of the world. So, the efficiency of delivering electrical power/energy has been a crucial concept for the direct current paradigm.

On the utilization side, again we can see the factor of efficiency making decisions. For example, we may say that electrical illumination which dates back to the incandescent bulb, invented by Edison, went on to fluorescent lights which gave higher efficiency and now LEDs providing the highest efficiency of all three technologies are being talked about. LEDs are inherently DC loads. Furthermore, heat-pump based heavy residential loads of space cooling and refrigeration are also witnessing the use of variable speed drives (VSDs) which provide higher efficiency as compared to the earlier On-Off control based compressors. VSDs use an intermediate DC stage and can be operated directly from DC provided a suitable voltage is available. Hence, DC is making a come-back in the system, and efficiency may be one of the determining factors.

In this research effort, we present a mathematical setup for the analysis of efficiency of a DC distribution system for use in a microgrid or otherwise. The paper presents a foundation level mathematical model that allows to maintain the individuality of each load in a house (as well as the whole system), the efficiency characteristic of an associated converter and the solar energy in each home in the model. Guidelines for using this model for efficiency studies will also be presented in the paper. The subsequent section will present a brief and relevant literature review as well as the gap in the present body of knowledge where this research effort aims to make its contribution.

\section{LITERATURE REVIEW}

As mentioned in the introduction, DC power distribution is currently in the research phase and a number of efforts have been directed towards it [10]-[17]. Dastgeer [18] is a recent review paper of the authors, related to efficiency analysis studies of DC distribution systems and microgrids (the current article, takes up the idea of mathematical modelling presented in [18]). Over here, we focus this section towards the review of system modelling used in efficiency studies of DC systems.

H. E. Gelani and F. Dastgeer [19] presents an efficiency study of DC distribution systems and performed a sensitivity analysis of the efficiency w.r.t loss coefficients of DC/DC system transformer. US Buildings Energy Data book was used for system modelling; wherein a single building block (BB) was taken to be composed of three categories of loads-natively DC, natively AC and independent (of AC or DC) loads. Various types of residential loads such as heating, cooling, and electronics. were actually categorized in these three types for the MATLAB based system simulation. Furthermore, US Energy Information Administration data was used to set daily load demand of a BB to be $30 \mathrm{kWh}$ and averaged power values were used (rather than instantaneous) for the modelling of the loads.

F. Dastgeer and H. E. Gelani [20] performs a comparative efficiency study for AC and DC systems. The modelling used is similar to that in [19] averaged load models (rather than instantaneously varying loads) are used and a single BB model is replicated on the whole system. Besides natively AC, natively DC and Independent load categories; a variable speed drive (VSD) loads category is also used in this MATLAB based simulation study-VSD category includes the loads of space heating and cooling.

B. Glasgo, I. L. Azevedo, and C. Hendrickson [3] is another simulation based comparison of DC and $\mathrm{AC}$ for residential power distribution. The authors have used data of Pecan Street Research Institute for modelling residential loads. Appliances were separated into five categories (Refrigeration, AC motors, Electric Vehicles, Resistance heating, others) and battery storage as well as solar energy were also included. Over here, again the bundling of appliances into categories leads to the loss of intricate variations of individual loads. Furthermore, similar to [19], [20] the use of a single PEC for a whole category of appliances is a departure from the real world scenario where a single converter is used for each appliance. However, as opposed to [19], [20], the individuality of homes is not lost and each home is a different entity in the simulation since the data comes from a real world source.

D. L. Gerber et al. [4] is a simulation based study for comparative efficiency study of DC and AC for commercial buildings. The studies are carried out for small and medium sized office buildings; results for large building have also been presented in appendix. As regards the system modelling, the authors have assumed all loads to be operating internally on DC. Furthermore, the building-to-building variation in loads is not mentioned explicitly. Similarly, the solar power and power electronic converters are apparently assumed to be the same at a given time in various buildings of the system. Moreover, the use of a single PEC for a whole category of appliances is again a departure from the real world scenario.

V. Vossos, K. Garbesi, and H. Shen [10] present a simulation based comparison of AC and DC power distribution for net-metered US residential buildings. For the DC system, the study categorizes all loads into three classes-Cooling, Non-cooling and low power loads. Furthermore, averaged loads models as well as averaged PEC efficiency values have been apparently used by the authors. The paper concludes that DC could be beneficial for US houses with net-metered PV systems; under certain conditions. 
H. R. Atia et al. [21] presents an efficiency analysis of AC and DC coupled microgrids considering load profile variations. The authors mention the use of a probabilistic model based on Queue theory for load profile estimation for residential buildings. Fixed values for PEC efficiencies were assumed in this study and details of individual appliances are not explicitly mentioned. The authors conclude AC system to be more efficient as compared to DC. Furthermore, in the relatively distant past, [14], [16] are a couple of efforts which discuss the comparative efficiency of DC and AC distribution systems- ([14] is simulation based). Instantaneous variations in loads have not been discussed in these efforts and the PEC efficiency values have also been assumed to be fixed-any detailed mathematical models have not been developed. Besides the aforementioned literature, [22]-[26] are other articles which touch upon DC power in one way or the other.

\subsection{The contribution of the current effort}

The current research effort presents a unique modeling in the sense that it allows for each load in the system to be treated as a distinguishable entity instead of being bundled/grouped into an appliance class/category. The modeling is detailed and intricate providing the capacity to include every single load of a building, with the provision to have a separate PEC (with its own efficiency characteristics) for the load. Furthermore, instead of making multiple copies/replicas of the same building block in a model, the current work allows each building to be a separate unit.

Moreover, as mentioned earlier in this section, various research efforts are based on simulation studies. A detailed mathematical model for the sake of system efficiency analysis is apparently missing in the current body of literature. The current work intends to present such a framework for the detailed efficiency analysis (and comparison) of a DC distribution system which maintains the individuality of each load in a house, the efficiency characteristics of each associated converter as well as the solar generation of the house. This is in contrast to those earlier efforts where modelling used averaging or merging different loads into categories/classes wherein the individuality of loads was lost. Moreover, in section 4 we present some guidelines for the use of our mathematical framework for future studies of DC network efficiency analyses.

\section{MODELLING FOR THE SAKE OF EFFICIENCY ANALYSIS}

In this section, we present the system modelling for the comparative efficiency analysis of the DC and AC distribution systems respectively.

\subsection{DC distribution system}

A detailed mechanical model for DC distribution system is presented in this section. Solid State Transformers (SST) will be replacing the conventional electromagnetic AC/AC transformer and the voltage level choice can depend on several factors. As in [18], [20], inside a single building block (BB), the loads are divided into four types-these are:

A Inherently AC loads (e.g. induction motor based clothes washer)

D Inherently DC loads (e.g. LED lights)

I Loads independent of power supply type (e.g. an electric iron can work with DC if suitable voltage is supplied)

VSD Variable speed drive loads (e.g. inverter type refrigerator)

Figure 1 presents a schematic diagram of a single building in the system. VSD loads have been assigned a separate category because of their special requirement of two stage power electronic conversion (AC/DC and then DC/AC) when used with the current AC power distribution systems. In the DC systems, these loads may add to system efficiency by omission of the first AC/DC stage if the line mains supplied DC voltage is directly useable by the loads for their second stage DC/AC conversion. Here, we assume this to be the case. If it is not so, then a DC/DC pre-conversion will be required before the second stage can take place.

Subsequently, four different multilayer load matrices for each of the four types of load are formed for all the BBs being served by the system SSTs. Here, the DC loads connected to all the SSTs have been represented in the given matrix. Where each layer of the matrix is showing the DC loads connected to a single SST and total SSTs range from ' $a$ ' to ' $x$ '.

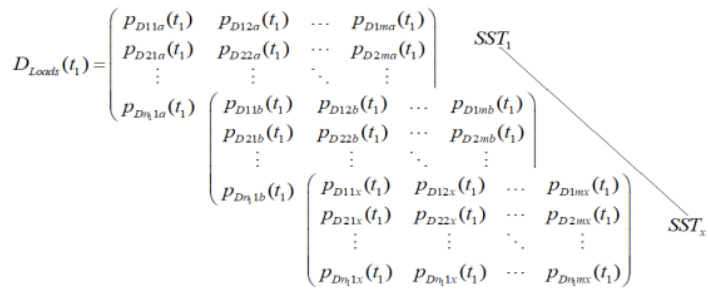


Here each column of a single layer is representing power demand of individual $D$ type loads in one BB-the loads range from ' $l$ ' to ' $n_{l}$ ' and BBs range from ' $l$ ' to ' $m$ '. For any BB with lesser than $n_{l}$ loads, the matrix entries may be put zero (with an adjustment in the software code being used for running the model, to avoid a divide by zero error). Thus $\mathrm{p}_{\mathrm{D} 11}\left(\mathrm{t}_{1}\right)$ shows the power demand of the first load in consideration of BB1 at time instant $t_{1}$. Because of the natural daily load variation, the study will need to be carried out at multiple instances of time throughout a day (and through various days of the year if seasonal variation is also to be included). The remaining three load matrices may be written similar to (1). Subsequently, the matrices for rated power of the various PECs which will be serving the loads are required. For the DC/DC converters, the matrix may be written as (2).

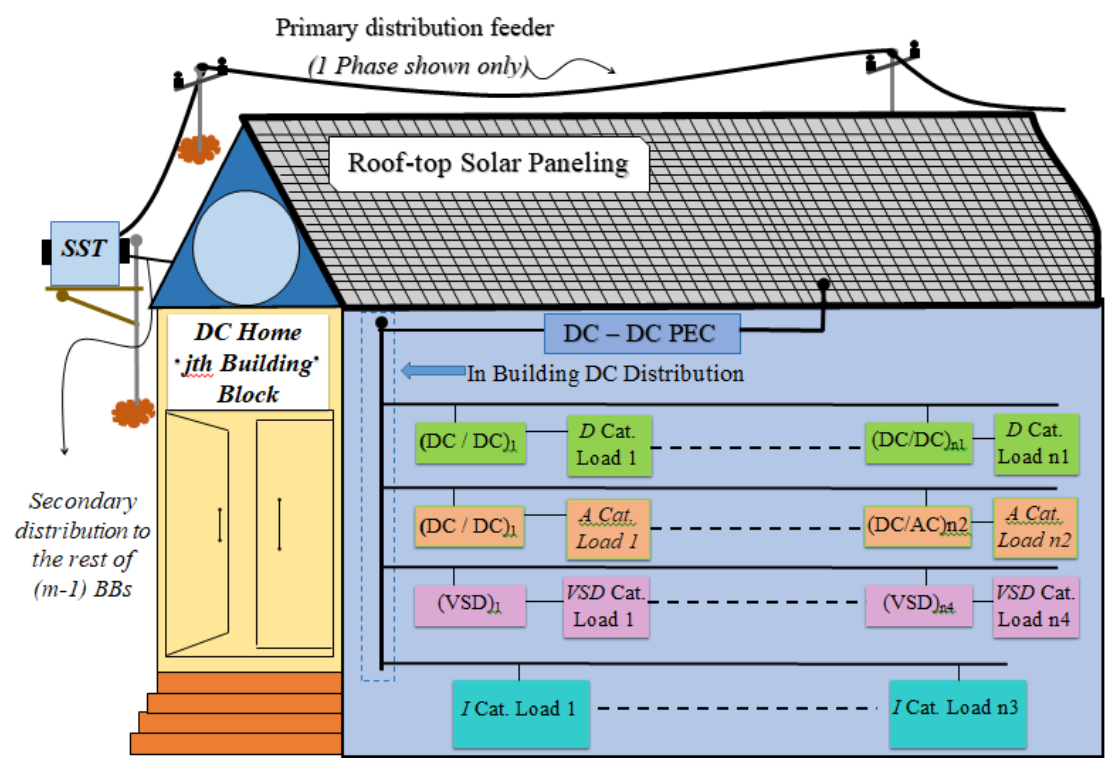

Figure 1. A schematic diagram of a single BB in the model

$$
d / d_{\text {rating }}=\left(\begin{array}{cccc}
r_{d d 11 a}\left(t_{1}\right) & r_{d d 12 a}\left(t_{1}\right) & \ldots & r_{d d 1 m a}\left(t_{1}\right) \\
r_{d d 12 a}\left(t_{1}\right) & r_{d d 22 a}\left(t_{1}\right) & \cdots & r_{d d 2 m a}\left(t_{1}\right) \\
\vdots & \vdots & \ddots & \vdots
\end{array}\right)
$$

Were $r_{d d i j k}$ represents the rated power of the PEC attached to the $i_{t h}$ DC load in the $j_{t h}$ building connected to kth SST. Similar matrices (of $d / a_{\text {rating }}$ and $v s d_{\text {rating }}$ ) will be created for the DC/AC inverters and the VSD load converters (with- or without a DC/DC pre-conversion as mentioned earlier) of the building. It is worth mentioning that the current modeling is assuming a worst case scenario of using PEC for each DC load of the system. For loads directly operable with the supplied DC voltage level, this conversion is inessential and the system model may be adjusted accordingly.

The next step is to determine the operating point of each load upon its efficiency characteristic; subsequent to which the input power of each load may be evaluated. Here, the efficiency characteristics are approximated to polynomial functions up to fourth degree. This may vary from case to case, and even the polynomial approximation may be unsuitable for efficiency curves showing high degree of variation-in such cases the model may be altered to involve higher polynomial degrees and piecewise functions respectively. Over here, we model an efficiency curve of, say the $i_{t h}$ DC/DC converter in the $j_{t h}$ building attached with kth SST as (3). 


$$
\eta_{d d-i j}\left(t_{1}\right)=a_{i j}\left(\frac{p_{D i j}\left(t_{1}\right)}{r_{d d i j}}\right)^{4}+b_{i j}(\ldots)^{3} c_{i j}(\ldots)^{2}+d_{i j}(\ldots)+e_{i j}
$$

The ratio $\left(p_{D i j k}\left(t_{1}\right) / r_{d d i j k}\right)$ is not written repeatedly for brevity. Furthermore, as the load $p_{D i j k}\left(t_{1}\right)$ is itself a function of time, hence the efficiency point of the corresponding DC/DC PEC becomes (an indirect) function of time. Although, strictly from the efficiency characteristic point of view $\eta_{d d-i j k}\left(t_{1}\right)$ is a (direct) function of converter loading. Prior to the execution of this step, the efficiency curves of the various PECs will be identified and the coefficients $a, b, c, d$ and e will be determined for each characteristic via curve fitting to generate $n 1 x m x k$ matrices for each coefficient. Similar is required for $\eta_{d a-i j k}$ and $\eta_{v s d-i j k}$. Subsequently, for the total load of a single BB (say $j_{t h}$ building), the input power $p_{j}\left(t_{l}\right)$ may be computed and a total sum of the summations of all four types of load categories eventually yields the total input power $p_{j}\left(t_{l}\right)$ demanded by the $j_{t h}$ building at time stamp $t_{1}$.

$$
\begin{aligned}
P_{j}\left(t_{1}\right)=\sum_{i=1}^{n_{1}} P_{D i j k-i n}\left(t_{1}\right)+\sum_{i=1}^{n_{2}} P_{A i j k-i n}\left(t_{1}\right)+\sum_{i=1}^{n_{3}} P_{I i j k-i n}\left(t_{1}\right)+\sum_{i=1}^{n_{4}} P_{V S D i j k-i n}\left(t_{1}\right) \\
=\sum_{i=1}^{n_{1}} \frac{P_{D i j k-i n}\left(t_{1}\right)}{\eta_{d d-i j k}}+\sum_{i=1}^{n_{2}} \frac{P_{A i j k-i n}\left(t_{1}\right)}{\eta_{d a-i j k}}+\sum_{i=1}^{n_{3}} P_{I i j k-i n}\left(t_{1}\right) \\
+\sum_{i=1}^{n_{1}} \frac{P_{V S D i j k-i n}\left(t_{1}\right)}{\eta_{d a-i j k}}
\end{aligned}
$$

Moreover, the modelling allows each home to have a separate PV system and the solar power produced in the $j_{\text {th }}$ building at time $t_{1}$ may be expressed as

$$
P_{\text {solar-j }}\left(t_{1}\right)=\left\{\lambda_{S D-j} \times\left[\alpha_{j}\left(t_{1}\right)^{4}+\beta_{j}\left(t_{1}\right)^{3}+\gamma_{j}\left(t_{1}\right)^{2}+\delta_{j}\left(t_{1}\right)^{1}+\varepsilon_{j}\left(t_{1}\right)\right]\right\}
$$

Again we have assumed a fourth degree polynomial for the curve fitting of solar generation (PV panel output) graphs and the coefficients $\alpha, \beta, \gamma, \delta$ and $\varepsilon$ may be different for each BB in the model i.e. each building may have a different solar capacity/panel orientation. Furthermore, the PV panel output may pass through different converters in different buildings, hence we introduce the variable $\lambda_{S D}$ which is a fractional factor representing PEC losses in conversion of solar power to the DC voltage useable by the building.

Furthermore, for an even detailed modeling, $\lambda_{S D}$ may itself be taken as a varying factor and expressed as a mathematical function of the power being generated; or its values (for a single building) may be stored in a look-up table, wherein the appropriate value will be used for the analysis time stamp in consideration. Subsequently, the total power demand of $j_{t h}$ building while ignoring conduction losses for secondary distribution (which may draw its support from [4] who assumed line losses to be comparable in $\mathrm{AC}$ and DC homes-the model needs augmentation to include these in a non-comparative study) may be expressed as (6).

$$
P_{\text {demand }-j}\left(t_{1}\right)=P_{j}\left(t_{1}\right)-P_{\text {solar }-j}\left(t_{1}\right)
$$

Subsequently, for the total load of a single SST (say ath SST), the output power pout-SSTa $_{1}$ ( ${ }_{1}$ ) may be computed by using the total summation of load demands of all buildings at time stamp $t_{l}$.

$$
P_{\text {out-SSTa }}\left(t_{1}\right)=\sum_{j=1}^{\mathrm{m}} P_{\text {demand }-j}\left(t_{1}\right)
$$

Subsequently, the input power drawn (or output power supplied in case of excess generation) by the system SST may be evaluated as (8).

$$
P_{\text {in-SSTa }}\left(t_{1}\right)=\frac{P_{\text {out }-S S T a}\left(t_{1}\right)}{\eta_{S S T a}\left(t_{1}\right)}
$$

Again the efficiency function of the SST $\left(\eta_{S S T}\right)$ may be treated as a polynomial expression; moreover, two different functions may be used for forward and reverse power flow. The conduction losses (in-building and SST-to-building) have not been taken into account in this model-they may be deemed ignorable in a DC vs. AC comparative efficiency study [20]. Again, in a non-comparative study, the model needs augmentation to include them. However, the conduction losses in primary distribution system from grid to single SST (say ath SST) can be modelled as (9).

$$
P_{\text {ll-SSTa }}\left(t_{1}\right)=\frac{P_{\text {in-SSTa }}\left(t_{1}\right)}{V_{\text {line }}\left(t_{1}\right)} \times P_{\text {line }-S S T a}
$$

Subsequently, for the total power drawn from DC grid can be calculated by adding the input power and conduction line losses of all the SSTs connected to grid as (10). 


$$
P_{D G}\left(t_{1}\right)=\sum_{k=a}^{x} P_{i n-S S T a}\left(t_{1}\right)-P_{l l-S S T a}\left(t_{1}\right)
$$

Subsequently, the total load demanded from grid can be calculate by the summations of load demands of all the categories (i.e. $D, A, I$ and $V S D$ ) for all the BBs connected to all the SSTs at time stamp $t_{l}$.

$$
\begin{aligned}
& P_{\text {Load-total }}\left(t_{1}\right)=\sum_{k=a}^{x} \sum_{j=1}^{m} \sum_{i=1}^{n_{1}} P_{\text {Dijk-in }}\left(t_{1}\right)+\sum_{\boldsymbol{d}_{k=a}}^{x} \sum_{j=1}^{m} \sum_{i=1}^{n_{1}} P_{\text {Aijk-in }}\left(t_{1}\right)+ \\
& \sum_{k=a}^{x} \sum_{j=1}^{m} \sum_{i=1}^{n_{1}} P_{\text {Iijk-in }}\left(t_{1}\right)+\sum_{k=a}^{x} \sum_{j=1}^{m} \sum_{i=1}^{n_{1}} P_{V S D i j k-i n}\left(t_{1}\right)
\end{aligned}
$$

Finally, the efficiency of the system under consideration may be evaluated as (12)

$$
\eta_{D C-D i s t r i b u t i o n}\left(t_{1}\right)=\frac{P_{\text {out }}\left(t_{1}\right)}{P_{\text {in }}\left(t_{1}\right)}=\frac{P_{\text {Load-total }}\left(t_{1}\right)}{P_{D G}\left(t_{1}\right)}
$$

where $p_{\text {Load-total }}\left(t_{l}\right)$ is the total load served in all the buildings and is a sum of the various loads present in $A_{\text {Loads }}\left(t_{1}\right), D_{\text {Loads }}\left(t_{1}\right), I_{\text {Loads }}\left(t_{1}\right)$ and $V S D_{\text {Loads }}\left(t_{1}\right)$ matrices. Note that efficiency is treated as a per unit value. (The efficiency results of (12) can be negative, refer to (6)). Subsequently, the energy efficiency of the system may be expressed as (13).

$$
\eta_{\text {Energy-DC-Distribution }}\left(t_{a}-t_{b}\right)=\frac{\sum_{t=1}^{\sigma} p_{\text {Load-total }}\left(t^{-}\right) \times t_{d u r}}{\sum_{t=1}^{\sigma} P_{D G}\left(t^{-}\right) \times t_{d u r}}
$$

Here $(t)^{-}$represents the value of a single discrete time sample in the duration $t_{a}-t_{b}$ during which

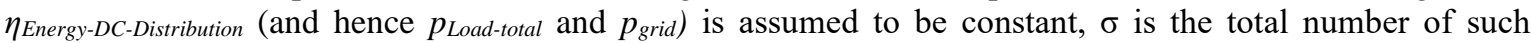
samples in the duration. $t_{\overline{d u r}}$ refers to the duration of the interval $t^{-}$. As shown in Figure 2 , a small error may

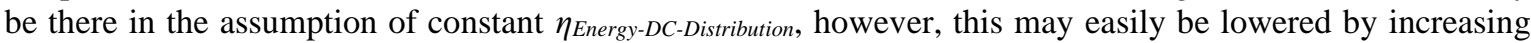
the number of samples of $\eta_{\text {Power-System }}$ and reducing the value of $\bar{t} \overline{d u r}$.

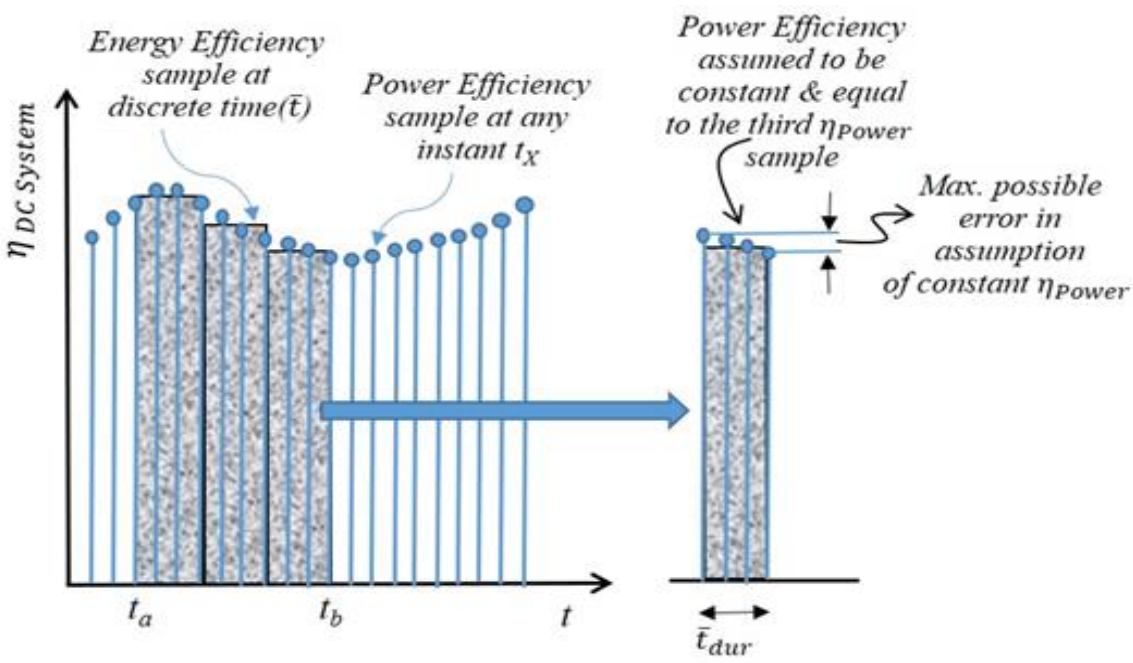

Figure 2. Power and energy efficiency

\subsection{AC distribution system}

For the AC distribution system efficiency study, the load matrix for D type loads may be represented as (14). Load matrices for categories $A, I$ and $V S D$ can also be represented similarly. Then instead of $D C / D C$ and $D C / A C$ converters of the $\mathrm{DC}$ system, this system will be using $A C / D C$ rectifiers and $A C / D C / A C$ converters for the VSD loads (note that here, we are not discussing the location of the PECs i.e. are the converters built-in for the loads or need to be provided externally-the current modelling is treating this topic as a general power electronic conversion). Subsequently, the matrices of $a / d_{\text {rating }}$ and $v s d_{\text {rating }}$ are required similar to the $d / d_{\text {rating, }} d / a_{\text {rating }}$ and $v s d_{\text {rating }}$ matrices used in the DC system. For $A C / D C$ converters, the matrix will be written as (15). 


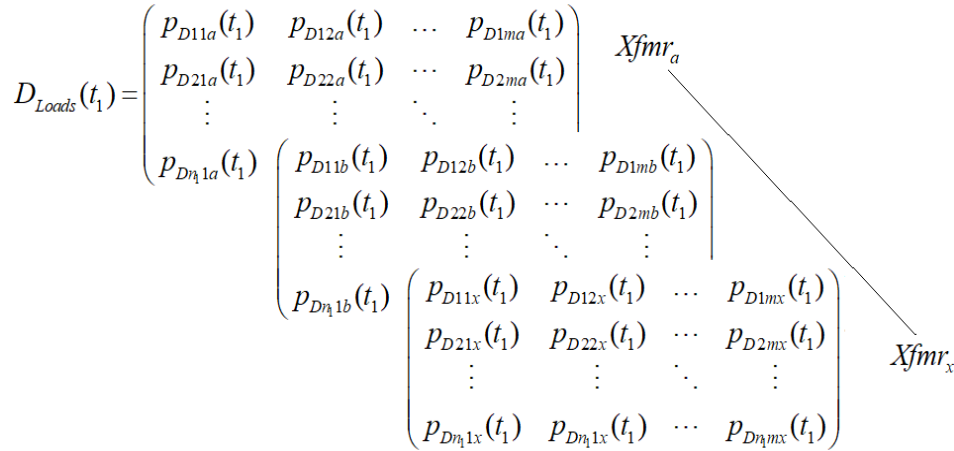

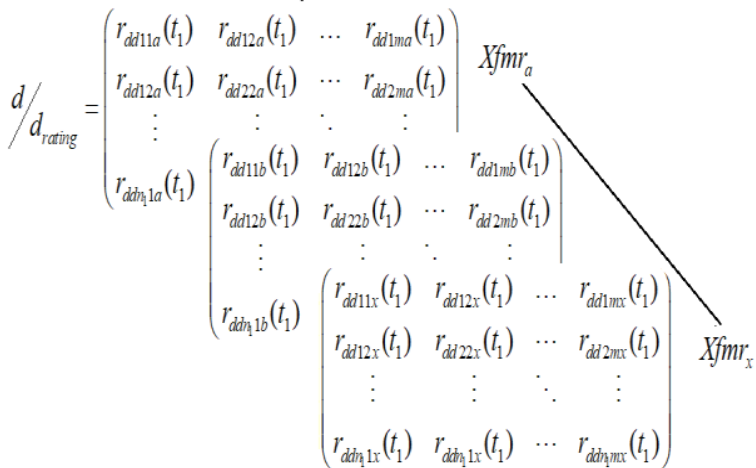

The $v s d_{\text {rating }}$ matrix will be different here (henceforth referred to as $v s d_{\text {rating }}$ ) because the power electronic conversion for $V S D$ loads in the $\mathrm{AC}$ system is composed of $A C / D C / A C$ conversion. Subsequently, the polynomial expressions for the efficiency of $D C / A C$ conversion $\left(\eta_{a d-i j k}\right)$ and the $A C / D C / A C$ conversion $\left(\eta^{`} V S D-i k j\right)$ of VSD loads may be written. Continuing the system modelling on similar lines as for the DC system, the total load power for $j$ th building is expressed as (16).

$$
\begin{aligned}
& \mathrm{P}\left(t_{1}\right)+Q_{j}\left(t_{1}\right)=\sum_{i=1}^{n_{1}} P_{D i j k-i n}\left(t_{1}\right)+\sum_{i=1}^{n_{2}}\left[P_{A i j k-i n}\left(t_{1}\right)+Q_{A i j k-i n}\left(t_{1}\right)\right]+\sum_{i=1}^{n_{3}} P_{I i j k-i n}\left(t_{1}\right)+ \\
& \sum_{i=1}^{n_{4}} P_{V S D i j k-i n}\left(t_{1}\right) \\
& =\sum_{i=1}^{n_{1}} \frac{P_{D i j k-i n}\left(t_{1}\right)}{\eta_{a d-i j k}}+\sum_{i=1}^{n_{2}}\left[P_{A i j k-i n}\left(t_{1}\right)+P_{A i j k-i n}\left(t_{1}\right)\right]+\sum_{i=1}^{n_{3}} P_{I i j k-i n}\left(t_{1}\right)+\sum_{i=1}^{n_{1}} \frac{P_{V S D i j k-i n}\left(t_{1}\right)}{\eta a_{d a-i j k}}
\end{aligned}
$$

Continuing on similar lines as earlier, the solar power generated by $j$ th building may be expressed as (17).

$$
P_{\text {solar }-j}\left(t_{1}\right)=\left\{\lambda_{S A-j} \times\left[\alpha_{j}\left(t_{1}\right)^{4}+\beta_{j}\left(t_{1}\right)^{3}+\gamma_{j}\left(t_{1}\right)^{2}+\delta_{j}\left(t_{1}\right)^{1}+\varepsilon_{j}\left(t_{1}\right)\right]\right\}
$$

Here the difference as compared to the DC model is the use of $\lambda_{S A}$ variable which represents solar to AC power conversion. Moving on the power drawn by the ath distribution transformer $p_{i n-X f m r}$ may be evaluated as (18).

$$
\begin{aligned}
& P_{\text {in-Xfmra }}\left(t_{1}\right)=\frac{\sum_{j=1}^{\mathrm{m}}\left[P_{\text {demand }-j}\left(t_{1}\right)+Q_{\text {demand }-j}\left(t_{1}\right)\right]}{\eta_{X f m r a}\left(t_{1}\right)} \\
& =\frac{\sum_{j=1}^{\mathrm{m}}\left[P_{j}\left(t_{1}\right)-P_{\text {Solar }-j}\left(t_{1}\right)\right]+\sum_{j=1}^{\mathrm{m}}\left[Q_{j}\left(t_{1}\right)\right]}{\eta_{X f m r a}\left(t_{1}\right)}
\end{aligned}
$$

Moreover, the equation for line losses of 3-phase AC Distribution system can be written as (19).

$$
P_{l l-X f m r a}\left(t_{1}\right)=I^{2} \times R_{\text {line-Xfmra }}=\frac{\mathrm{S}\left(t_{1}\right)}{3 \times R_{\text {line }}\left(t_{1}\right)}
$$

Here, $R_{\text {line-Xfmr }}$ is the AC resistance of all the three conductors used for power distribution from AC grid to Distribution transformer and $V_{\text {line }}$ is representing line to line voltages. Similarly, the total power drawn from AC Grid can be expressed as (20).

$$
S_{A G}\left(t_{1}\right)=P_{A G}\left(t_{1}\right)+Q_{A G}\left(t_{1}\right)=\sum_{K=a}^{\mathrm{x}}\left[P_{i n-X f m r k}\left(t_{1}\right)-P_{l l-X f m r k}\left(t_{1}\right)\right]+\sum_{K=a}^{\mathrm{X}} Q_{i n-X f m r k}\left(t_{1}\right)
$$


Finally, the system power and energy efficiency values may be determined via;

$$
\begin{aligned}
& \eta_{\text {AC-Distribution }}\left(t_{1}\right)=\frac{P_{\text {out }}\left(t_{1}\right)}{P_{\text {in }}\left(t_{1}\right)}=\frac{P_{\text {Load-total }}\left(t_{1}\right)}{P_{A G}\left(t_{1}\right)} \\
& \eta_{\text {Energy-AC-Distribution }}\left(t_{a}-t_{b}\right)=\frac{\sum_{t=1}^{\sigma} p_{\text {Load-total }}\left(t^{-}\right) \times t_{d u r}}{\sum_{t=1}^{\sigma} P_{A G}\left(t^{-}\right) \times t_{d u r}}
\end{aligned}
$$

\section{GUIDELINES FOR USING THE PRESENTED MODEL}

In this section we present some guidelines for using the proposed framework in comparative efficiency studies of DC and AC networks. Figure 3 presents a flow chart showing the process steps for the DC system efficiency study, a similar chart may be made for the AC system. The first three steps are required to initiate the study, then 4-8 may be continued in a loop for various time samples $t_{1}, t_{2}, \ldots$, of a duration $t_{a}-t_{b}$. Subsequently, the energy efficiency may be evaluated using the stored values of pLoad-mBBs and pin-SST.

Furthermore, an efficiency analysis using our framework will need to derive load data from datamining or non-intrusive load monitoring (NILM) tools and build the required data set which will also include PEC characteristics and solar generation of each home. Nevertheless, it should be mentioned that although this model presents a framework for an efficiency analysis incorporating the individuality of various system elements, still, a future study that aims to use this model can make some assumptions/repetitions that digress from this individuality based upon the availability of data, the duplication of loads in physical residential buildings as well as the level of depth and details required by the study.

Moreover, the framework may be used purposes other than the evaluation of efficiency values of AC and DC systems. For example, as mentioned in [18], sensitivity analysis is an idea for the efficiency studies and the proposed framework can be used for exploration in this direction. Furthermore, it is worth mentioning that with substantial solar generation installed/assumed in buildings, there may be times where efficiency calculations in DC and AC systems yield negative values indicating reverse flow of power/energy. In this case a higher negative value (in other words a smaller absolute value) indicates better system performance.

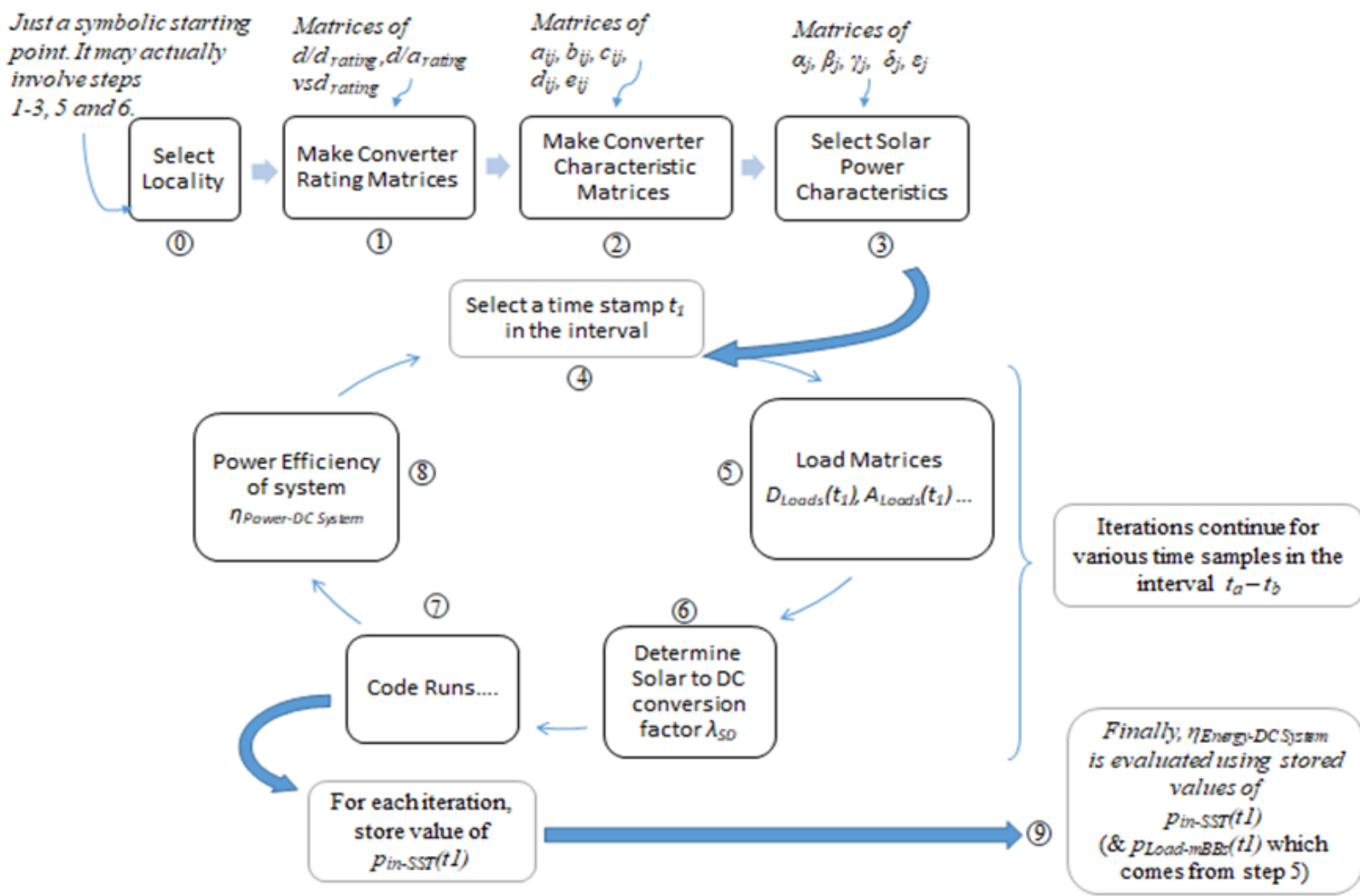

Figure 3. Brief flow chart for DC system efficiency study-step 6 may be incorporated as part of step 7 


\section{SHORTCOMINGS AND FUTURE} assumed that;

Lastly, we list some of the assumptions which have not been removed in this framework. It has been

- $\quad$ The reactive power requirement from inverters is met entirely by their output filter, and has no effect on input DC side. Inverter efficiency is solely dependent on output real power.

- $\quad$ Rectifiers require no input reactive vars as perfect power factor correction is assumed.

- $\quad$ VSD loads require no DC/DC pre-conversion and can directly work with the DC voltage level in DC system.

- Conduction losses have been ignored.

Working with these assumptions may be possible future work directions. Especially, primary distribution system line-losses should be included. Although only real power will be used for power efficiency analysis. However, a proper inclusion of these calls for usage of load-flow analysis iterative techniques such as DC flow or Newton-Raphson power flow, and since the effect of line losses on comparative analyses is minor, hence they have been left for a possible future expansion.

\section{CONCLUSION}

This paper has presented a mathematical modelling based test setup for the efficiency studies of DC distribution networks and microgrids-especially for comparison with counterpart AC systems. It may be said that besides presenting a mathematical model for the efficiency studies of DC networks; avoiding the averaging of loads and the merging of loads into categories that deprives them of their individuality has been an aim of this study. Nevertheless, we could not make it an assumption-free framework. This may be worked upon by a future study which utilizes this model as it may choose which assumption to keep and which to replace by a closer to real world augmentation.

\section{ACKNOWLEDGEMENTS}

Acknowledgment is made to the work of the three final year project students Syed Ahmed Ali, Abdullah Bilal and Hafiz Muhammad Waqas whose project has helped to adjust the framework presented in this paper.

\section{REFERENCES}

[1] C. L. Sulzberger, "Triumph of AC - from Pearl Street to Niagara," IEEE Power and Energy Magazine, vol. 1, no. 3, pp. 64-67, 2003, doi: 10.1109/MPAE.2003.1197918.

[2] C. L. Sulzberger, "Triumph of AC. 2. The battle of the currents," IEEE Power and Energy Magazine, vol. 1, no. 4, pp. 70-73, 2003, doi: 10.1109/MPAE.2003.1213534.

[3] B. Glasgo, I. L. Azevedo, and C. Hendrickson, "How much electricity can we save by using direct current circuits in homes? Understanding the potential for electricity savings and assessing feasibility of a transition towards DC powered buildings," Applied Energy, vol. 180, pp. 66-75, 2016, doi: 10.1016/j.apenergy.2016.07.036.

[4] D. L. Gerber, V. Vossos, W. Feng, C. Marnay, B. Nordman, and R. Brown, "A simulation-based efficiency comparison of AC and DC power distribution networks in commercial buildings," Applied Energy, vol. 210, pp. 1167-1187, 2017, doi: 10.1016/j.apenergy.2017.05.179.

[5] W. Yang, A. Zhang, S. Song, H. Zhang, and J. Wang, "Comparative Study on Radial Topology 10kV AC and \pm $10 \mathrm{kV}$ DC," 2016 Chinese Control and Decision Conference (CCDC), 2016, pp. 6216-6221, doi: 10.1109/CCDC.2016.7532116.

[6] R. Farooq, L. Mateen, M. Ahmad, S. Q. Akbar, H. A. Khan, and N. A. Zaffar, "Smart DC microgrids: Modeling and power flow analysis of a DC Microgrid for off-grid and weak-grid connected communities," 2014 IEEE PES Asia-Pacific Power and Energy Engineering Conference (APPEEC), 2014, pp. 1-6, doi: 10.1109/APPEEC.2014.7066139.

[7] F. Wang, Z. Lei, X. Xu, and X. Shu, "Topology Deduction and Analysis of Voltage Balancers for DC Microgrid," IEEE Journal of Emerging and Selected Topics in Power Electronics, vol. 5, no. 2, pp. 672-680, 2017, doi: 10.1109/JESTPE.2016.2638959.

[8] R. Mohanty and A. K. Pradhan, "Protection of Smart DC Microgrid with Ring Configuration using Parameter Estimation Approach," IEEE Transactions on Smart Grid, vol. 9, no. 6, pp. 6328-6337, 2017, doi: 10.1109/TSG.2017.2708743

[9] Q. Xu, X. Hu, P. Wang et al., "A Decentralized Dynamic Power Sharing Strategy for Hybrid Energy Storage System in Autonomous DC Microgrid," IEEE Transactions on Industrial Electronics, vol. 64, no. 7, pp. 59305941, 2017, doi: 10.1109/TIE.2016.2608880.

[10] V. Vossos, K. Garbesi, and H. Shen, "Energy savings from direct-DC in U.S. residential buildings," Energy and Buildings, vol. 68, no. Part A, pp. 223-231, 2014, doi: 10.1016/j.enbuild.2013.09.009

[11] Z. Liu and M. Li, "esearch on Energy Efficiency of DC Distribution System," AASRI Procedia, vol. 7, pp. 68-74, 2014, doi: 10.1016/j.aasri.2014.05.031. 
[12] R. Weiss, L. Ott, and U. Boeke, "Energy efficient low-voltage DC-grids for commercial buildings," 2015 IEEE First International Conference on DC Microgrids (ICDCM), 2015, pp. 154-158, doi: 10.1109/ICDCM.2015.7152030.

[13] U. Boeke and M. Wendt, "DC power grids for buildings," 2015 IEEE First International Conference on DC Microgrids (ICDCM), 2015, pp. 210-214, doi: 10.1109/ICDCM.2015.7152040.

[14] F. Dastgeer and A. Kalam, "Efficiency comparison of DC and AC distribution systems for distributed generation," 2009 Australasian Universities Power Engineering Conference, 2009, pp. 1-5.

[15] M. Starke, L. M. Tolbert, and B. Ozpineci, "AC vs. DC distribution: A loss comparison," 2008 IEEE/PES Transmission and Distribution Conference and Exposition, 2008, pp. 1-7, doi: 10.1109/TDC.2008.4517256.

[16] D. J. Hammerstrom, “AC Versus DC Distribution Systems-Did We Get it Right?," 2007 IEEE Power Engineering Society General Meeting, 2007, pp. 1-5, doi: 10.1109/PES.2007.386130.

[17] A. Sannino, G. Postiglione, and M. H. J. Bollen, "Feasibility of a DC network for commercial facilities," IEEE Transactions on Industry Applications, vol. 39, no. 5, pp. 1499-1507, 2003, doi: 10.1109/TIA.2003.816517.

[18] F. Dastgeer, H. E. Gelani, H. M. Anees, Z. J. Paracha, and A. Kalam, "Analyses of efficiency/energy-savings of DC power distribution systems/microgrids: Past, present and future," International Journal of Electrical Power \& Energy Systems, vol. 104, pp. 89-100, 2019, doi: 10.1016/j.ijepes.2018.06.057.

[19] H. E. Gelani and F. Dastgeer, "Efficiency analyses of a DC residential power distribution system for the modern home," Advances in Electrical and Computer Engineering, vol. 15, no. 1, pp. 135-142, 2015, doi: 10.4316/AECE.2015.01018.

[20] F. Dastgeer and H. E. Gelani, "A Comparative analysis of system efficiency for AC and DC residential power distribution paradigms," Energy and Buildings, vol. 138, pp. 648-654, 2017, doi: 10.1016/j.enbuild.2016.12.077.

[21] Atia, A. Shakya, P. Tandukar, U. Tamrakar, T. M. Hansen, and R. Tonkoski, "Efficiency analysis of AC coupled and DC coupled microgrids considering load profile variations," 2016 IEEE International Conference on Electro Information Technology (EIT), 2016, pp. 695-699, doi: 10.1109/EIT.2016.7535324.

[22] S. H. Chew et al., "Design considerations for ELVDC power distribution system integrated directly with DC energy sources for LED lighting in high-rise built environment," 2015 IEEE International Telecommunications Energy Conference (INTELEC), 2015, pp. 1-6, doi: 10.1109/INTLEC.2015.7572300.

[23] D. Fregosi et al., "A comparative study of DC and AC microgrids in commercial buildings across different climates and operating profiles," 2015 IEEE First International Conference on DC Microgrids (ICDCM), 2015, pp. 159164, doi: 10.1109/ICDCM.2015.7152031.

[24] U. Manandhar, A. Ukil and T. K. Kiat Jonathan, "Efficiency comparison of DC and AC microgrid," 2015 IEEE Innovative Smart Grid Technologies - Asia (ISGT ASIA), 2015, pp. 1-6, doi: 10.1109/ISGT-Asia.2015.7387051.

[25] T. Dragicevic, J. C. Vasquez, J. M. Guerrero and D. Skrlec, "Advanced LVDC Electrical Power Architectures and Microgrids: A step toward a new generation of power distribution networks," IEEE Electrification Magazine, vol. 2, no. 1, pp. 54-65, March 2014, doi: 10.1109/MELE.2013.2297033.

[26] Ahmed T. Elsayed, Ahmed A. Mohamed, Osama A. Mohammed, "DC microgrids and distribution systems: An overview," Electric Power Systems Research, vol. 119, 2015, pp. 407-417, doi: 10.1016/j.epsr.2014.10.017.

\section{BIOGRAPHIES OF AUTHORS}

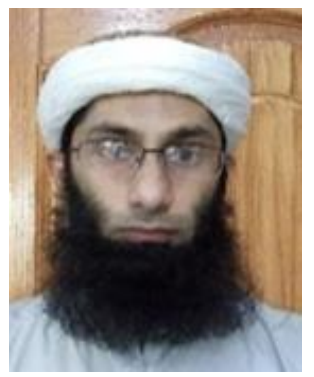

Faizan Dastgeer is currently serving in the capacity of Assistant Professor at the department of Electrical Engineering, University of Engineering \& Technology (UET) Lahore - Faisalabad campus. Previously, he completed his B.Sc. Electrical Engineering with specialization in power (engineering) from UET Lahore in the year 2007; and went on to become a member of the degree of Doctorate of Philosophy of Victoria University Melbourne, in the year 2012. His research interest has been focused towards the efficiency of DC power distribution systems and he is the author of about half a dozen journal papers in this field. Dr. Dastgeer is a registered member of the Pakistan Engineering Council.

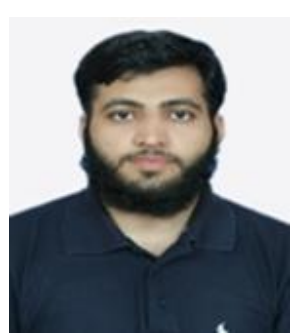

Hafiz Muhammad Anees (MS Fellow - JE Officer) was born in Lahore, Punjab, Pakistan in 1993. He is currently working in Pakistan's renowned organization and pursuing MS from Pakistan Institute of Engineering and Applied Sciences (PIEAS), Islamabad, Pakistan. He completed his first MS degree in Electrical Engineering with the specialization in Power from the Pakistan's prestigious institute U.S. - Pak. Center for Advanced Studies in Energy (USPCAS-E) NUST H-12, Islamabad in 2020. In 2017, he graduated in Electrical Engineering (Power) from University of Engineering and Technology (UET) Lahore. His current research field is Microgrids, Multi-Microgrids and Smartgrids and one of his research publications received "The Best International Innovative Research Award, 2020". The mathematical modeling of Multi-Microgrids is one of his major speciality in research. 


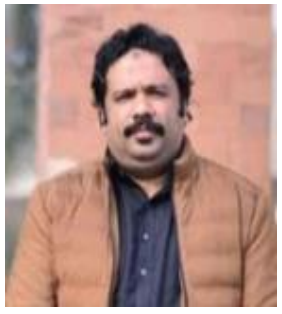

Dr Gelani received his BS, MS and Phd degrees in Electrical Engineering from University of Engineering and Technology Lahore Pakistan in 2009, 2014 and 2019 respectively. He is currently serving in the same university as assistant professor. He has supervised several projects related to electric power and energy. His research interests include efficiency analyses of AC and DC power distribution systems, DC microgrids and distributed generation.

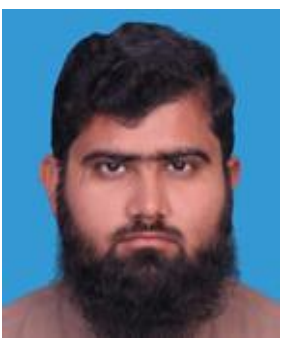

Kashif Amjad received the B.S. degree in electrical engineering with the specialization in power from the University of Engineering and Technology, Lahore in 2018. He is working in "Power and Renewable Energy Systems Lab" as a Lab Engineer in the Department of Electrical Engineering at National University of Computer and Emerging Sciences. Moreover, he is also pursuing the Master's degree in the same institute. His research interest includes power and renewable energy systems

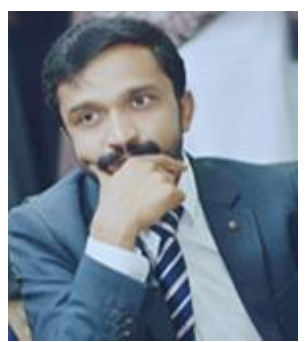

M. Rameez Javed was born in Sargodha, Punjab, Pakistan in 1989. He received BSc degree in Electronics Engineering from University of Engineering and Technology Taxila in 2012. He received his MSc degree in Electrical Engineering from UET Taxila with Gold Medal in 2016.

He served as Lecturer in University of Sargodha Engineering College (2016-2017). He is currently doing job as Lecturer in UET Lahore FSD campus. He is expert in Power Electronics, Smart Grid, Power system Protection and Power System Operation and control. 